\title{
Suboptimal Decentralized Controller Design for Chain Structures: Applications to Vehicle Formations
}

\author{
Assad Al Alam, Ather Gattami and Karl H. Johansson
}

\begin{abstract}
We consider suboptimal decentralized controller design for subsystems with interconnected dynamics and cost functions. A systematic design methodology is presented over the class of linear quadratic regulators (LQR) for chain graphs. The methodology is evaluated on heavy duty vehicle platooning with physical constraints. A simulation and frequency analysis is performed. The results show that the decentralized controller gives good tracking performance and a robust system. We also show that the design methodology produces a string stable system for an arbitrary number of vehicles in the platoon, if the vehicle configurations and the $L Q R$ weighting parameters are identical for the considered subsystems.
\end{abstract}

\section{INTRODUCTION}

The systems to be controlled are in many application domains getting larger and more complex. When there is interconnection between different dynamics, conventional optimal control algorithms provide a solution where full state information is required. However, it is often preferable and sometimes necessary to have a decentralized controller structure, since in many practical problems, the physical or communication constraints often impose a specific interconnection structure. Hence, it is interesting to design decentralized feedback controllers for systems of a certain structure and examine their overall performance.

The control problem and methodology in this paper is motivated by systems involving a chain of closely spaced heavy duty vehicles (HDVs), generally referred to as vehicle platooning. Through commercially available systems, for example radar and wireless communication, each vehicle is able to measure or receive the relative distance, relative velocity, and additional relevant information concerning the preceding vehicle. The objective is to maintain a predefined headway to the vehicle ahead. By traveling at a close distance to a HDV, the air drag is reduced [1]. Hence, the effort needed to maintain the desired relative velocity varies with the relative distance. This creates a coupling of the dynamics between vehicles throughout the platoon. In [2] it was shown that a fuel reduction of $4.7-7.7 \%$ can be experimentally obtained by utilizing the air drag reduction that occurs when driving HDVs closely spaced. However, due to the additional control effort produced by the existing

This work was supported by Scania CV AB, VINNOVA - FFI, and the Swedish Research Council.

A. Alam is with Research and Development, Scania CV AB, 15187 Södertälje, Sweden assad.alamescania.com

A. Gattami and Karl H. Johansson are with Electrical Engineering, ACCESS Linnaeus Centre, Royal Institute of Technology, 10044 Stockholm, Sweden gattami@kth.se, kallej@ee.kth.se control systems for maintaining the relative distance, the fuel consumption increases. Hence, it is of vast interest for the industry to produce a new fuel optimal control input. Considering the physical constraints in radio, it cannot be assumed that state information is available at every instance in time. Thus, a decentralized control strategy is crucial for practical implementation.

The problem of decentralized control has a long history. Team decision problems were introduced in [3], where each team member is trying to optimize a common cost function through limited information concerning the global state of nature. In [4], decentralized control was studied through a sequential manner by closing one loop at a time and in, [5], [6], under the assumption of spatial invariance. Decentralized control design for stability based on local model knowledge appeared in [7]. Control for chain structures in the context of platoons has been studied through various perspectives, e.g., [8]-[14]. It has been shown that control strategies may vary depending on the available information within the platoon. Maintaining a suitable relative distance, stability and robustness of the platoon have been identified to be amongst the main criteria to be considered. However, control design for platooning applications have mainly been ad hoc by tuning the control parameters. In [15]-[17], optimized procedures were presented to give a systematic approach to the design. However, the vehicle coupling was only introduced through the cost function. In contrast, we present a control algorithm that considers decentralized optimal control based upon systems with interconnected dynamics and local state information. For HDV platooning applications, the coupling is induced by the variation in aerodynamics between the vehicles and the state information is restricted due to information only being available from the immediate preceding vehicle. The former is essential in the analysis of fuel reduction potential for HDVs.

In this work, we are primarily concerned with the case of forming a decentralized control, solely based on local model knowledge, over the class of linear quadratic regulators (LQR) for chain structured interconnection graphs. However, the control design procedure can be generalized to the extent of acyclic graphs. We propose a systematic method to derive suboptimal stabilizing decentralized controllers, which imposes a lower block-diagonal structure on the feedback gain matrix. We also show that the controller gives a stable and robust closed-loop system.

The main contribution of this paper is to design an LQR- 
based method for deriving a suboptimal decentralized feedback that takes dynamic coupling into consideration. We give physical insight of how to derive the weighting factors for a specific problem with a chain structure. We also investigate the performance of the proposed controllers, under normal operating conditions, with respect to physical constraints that are imposed in a practical set-up. The structure of the controller feedback matrix can be tailored with respect to the locally available state information.

The outline of the paper is as follows. First we give a general description of the system structure and present the methodology to produce a systematic decentralized control design in Sec. II. We apply the methodology to the example of HDV platooning controller design in Sec. III, for which we give a physical interpretation of how to design the weighting parameters. Then we present the performance by giving a frequency analysis and simulation results in Sec. IV. Finally, in Sec. VI we present a give summary of the results and conclusions.

\section{The Structured LQR Problem}

In this section, we present the system under consideration and provide the general procedure for solving the suboptimal stabilizing decentralized controllers for interconnected systems with block tridiagonal structures. The method will be utilized for solving local state feedback control problems.

The objective of this study is to design suboptimal stabilizing decentralized controllers, solely based on local model knowledge, for a $N$-vehicle system when there is interconnection between different subsystem dynamics, without compromising system performance. The vehicles can only receive information from the interconnected preceding vehicle, which is generally the case for commercially available systems. However, the local information can naturally be extended to a larger subset.

Consider a system consisting of $N$ vehicles as depicted in Fig. 1, where each vehicle is interconnected with the preceding vehicle. The system structure under consideration can generally be given as

$$
\begin{array}{r}
\dot{x}=\left[\begin{array}{ccccc}
A_{11} & 0 & 0 & \ldots & 0 \\
A_{21} & A_{22} & 0 & \ldots & 0 \\
0 & A_{32} & A_{33} & \ldots & 0 \\
\vdots & \vdots & \vdots & \ddots & \vdots \\
0 & 0 & 0 & \ldots & A_{N N}
\end{array}\right]\left[\begin{array}{c}
x_{1} \\
x_{2} \\
x_{3} \\
\vdots \\
x_{N}
\end{array}\right] \\
+\left[\begin{array}{ccccc}
B_{1} & 0 & 0 & \ldots & 0 \\
0 & B_{2} & 0 & \ldots & 0 \\
0 & 0 & B_{3} & \ldots & 0 \\
\vdots & \vdots & \vdots & \ddots & \vdots \\
0 & 0 & 0 & \ldots & B_{N}
\end{array}\right]\left[\begin{array}{c}
u_{1} \\
u_{2} \\
u_{3} \\
\vdots \\
u_{N}
\end{array}\right],
\end{array}
$$

where $A_{i j}, i \neq j$, denotes the interconnection between the system dynamics. An optimal LQR state-feedback controller can be derived by minimizing the quadratic cost function given by

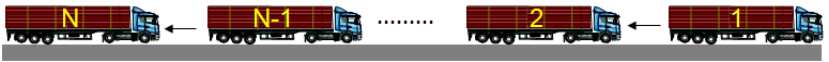

Fig. 1. The figure shows a platoon of $N$ heavy duty vehicles, where each is only able to communicate with the preceding vehicle.

$$
J(u)=x\left(t_{f}\right)^{T} S x\left(t_{f}\right)+\int_{t_{0}}^{t_{f}} x(t)^{T} Q x(t)+u(t)^{T} R u(t) d t
$$

where $S$ and $Q$ are positive semidefinite and $R$ is positive definite. The control input that minimizes (2) is given by

$$
\begin{aligned}
\dot{P} & =P B R^{-1} B^{T} P-A^{T} P-P A-Q \\
L & =R^{-1} B^{T} P \\
u_{\text {cen }}^{*} & =-L x
\end{aligned}
$$

where the optimal control solution, $u_{\mathrm{cen}}^{*}$, has a communication topology that provides each vehicle with full state information. However, such an assumption is not realistic, since the available information is limited in practice.

\section{Structural Decomposition}

Due to the constraints set upon the information topology and the interconnection between the subsystems (Fig. 1), the global system (1) can be divided into sub-blocks. Thereby, the first vehicle (subsystem 1) can optimize its control input by setting its weighting parameters $Q_{1}$ and $R_{1}$ with respect to the desired performance criterion. By conveying the information, each interconnected follower vehicle (subsystem $i$ ) can subsequently derive locally optimal stabilizing controllers based on the local model. The local optimization is performed separately for each vehicle and the weighting parameters $Q_{i}$ and $R_{i}$ in the respective optimization steps are set with respect to each vehicle's performance criteria. Therefore, they need only be known to the individual vehicle and the dimension can vary based upon the available state information. The matrix $Q_{i}$ will in particular have a specific form, which will contribute to the desired coupling behavior of the interconnected vehicle. As a result of subsequently deriving controllers based upon local model information and interconnection, a global suboptimal decentralized feedback matrix with a lower block diagonal form is produced with respect to (1), which can be given as

$$
L=\left[\begin{array}{ccccc}
L_{11} & 0 & 0 & \ldots & 0 \\
L_{21} & L_{22} & 0 & \ldots & 0 \\
0 & L_{31} & L_{33} & \ldots & 0 \\
\vdots & \vdots & \vdots & \ddots & \vdots \\
0 & 0 & 0 & \ldots & L_{N N}
\end{array}\right]
$$

Thus, a systematic decentralized LQR-optimization can be performed for each subsystem, as described in Algorithm 1. 


\section{Algorithm 1:}

0 ) Set the weight matrices $Q_{i}, R_{i}, i=1, \ldots, N$, positive definite and in accordance with the desired performance criteria.

1) Derive the locally optimal feedback controller, $u_{1}^{*}$, for subsystem 1 (the lead vehicle) by solving

$$
\begin{aligned}
& \min _{u_{1}} \int_{t_{0}}^{t_{f}} x_{1}^{T} Q_{1} x_{1}+u_{1}^{T} R_{1} u_{1} d t \\
& \text { s. t. } \dot{x}_{1}=A_{11} x_{1}+B_{1} u_{1} .
\end{aligned}
$$

$$
\begin{aligned}
\dot{P}_{1} & =P_{1} B_{1} R_{1}^{-1} B_{1}^{T} P_{1}-A_{11}^{T} P_{1}-P_{1} A_{11}-Q_{1}, \\
L_{11} & =R_{1}^{-1} B_{1}^{T} P_{1} \\
u_{1}^{*} & =-L_{11} x_{1} .
\end{aligned}
$$

2) Each preceding vehicle's dynamics is known to the follower vehicle. Therefore, utilize this information of subsystem $i-1$ in the control design of subsystem $i$ (the follower vehicle) and subsequently compute for $i=2, \ldots, N$,

$$
\begin{gathered}
\min _{u_{i}} \int_{t_{0}}^{t_{f}}\left[\begin{array}{c}
x_{i-1} \\
x_{i}
\end{array}\right]^{T} Q_{i}\left[\begin{array}{c}
x_{i-1} \\
x_{i}
\end{array}\right]+u_{i}^{T} R_{i} u_{i} d t \\
\text { s. t. } \\
{\left[\begin{array}{c}
\dot{x}_{i-1} \\
\dot{x}_{i}
\end{array}\right]=\underbrace{\left[\begin{array}{cc}
A_{(i-1)(i-1)}-B_{i-1} L_{i-1} & 0 \\
A_{i(i-1)} & A_{i i}
\end{array}\right]}_{\bar{A}_{i i}}\left[\begin{array}{c}
x_{i-1} \\
x_{i}
\end{array}\right]} \\
+\underbrace{\left[\begin{array}{c}
0 \\
B_{i}
\end{array}\right]}_{\bar{B}_{i}} u_{i},
\end{gathered}
$$

Obtain locally optimal, $u_{i}^{*}$, feedback by solving

$$
\begin{aligned}
\dot{P}_{i} & =P_{i} \bar{B}_{i} R_{i}^{-1} \bar{B}_{i}^{T} P_{i}-\bar{A}_{i i}^{T} P_{i}-P_{i} \bar{A}_{i i}-Q_{i}, \\
\tilde{L}_{i} & =R_{i}^{-1} \bar{B}_{i}^{T} P_{i}, \\
u_{i}^{*} & =-\tilde{L}_{i}\left[\begin{array}{c}
x_{i-1} \\
x_{i}
\end{array}\right]
\end{aligned}
$$

where $\tilde{L}_{i}=\left[\begin{array}{ll}L_{i(i-1)} & L_{i i}\end{array}\right]$.

Theorem 1: Consider a chain of $N$ interconnected subsystems with dynamics given by (1). Algorithm 1 provides a locally optimal state-feedback controller $u=-L x$ with $L$ as in (3) that results in a globally asymptotically stable closed-loop system.

Proof: Consider subsystems $\left(\bar{A}_{i i}, \bar{B}_{i}\right), i=1, \ldots, N$, as introduced in Algorithm 1. It is easy to see that with the specified state-feedback control law $u=-L x$ the resulting closed-loop system has eigenvalues given as the solutions to

$$
\prod_{i=1}^{N} \operatorname{det}\left[\lambda I-\left(A_{i i}-B_{i} L_{i i}\right)\right]=0 .
$$

Thus, Algorithm 1 produces a globally asymptotic stable system, since

$$
\operatorname{Re}\left[\lambda_{i}\left(A_{i i}-B_{i} L_{i i}\right)\right]<0, \forall i .
$$

\section{Application to Heavy Duty Vehicle PLATOONING}

In this section, we consider the problem that inspired the control design procedure over the class of LQR control for chain structured interconnection graphs (Fig. 1). We also investigate the performance with respect to system requirements and stability.

The state equation of a single HDV is [18],

$$
\begin{aligned}
\dot{s}=v & \\
m_{t} \dot{v}= & F_{\text {engine }}-F_{\text {brake }}-F_{\text {airdrag }}(v) \\
& \quad-F_{\text {roll }}(\alpha)-F_{\text {gravity }}(\alpha) \\
= & k_{e} T_{e}-k_{b} F_{\text {brake }}-k_{d} v^{2} \\
& \quad-k_{f r} \cos \alpha-k_{g} \sin \alpha
\end{aligned}
$$

where $v$ is the vehicle velocity, $m_{t}$ denotes the accelerated mass and $T_{e} \in \mathbb{R}$ denotes the net engine torque. $k_{e}, k_{b}, k_{d}, k_{f r}$, and $k_{g}$ denote the characteristic vehicle and environment coefficients for the brake, air drag, road friction, and gravitation respectively.

The non-linear model, (4), can be linearized with respect to a set reference velocity, an engine torque which maintains the velocity, a fixed time gap between the vehicles, and a constant slope.

When traveling in a platoon, the air drag has a significant impact on the overall resistive forces, which is one of the key factors in fuel reduction possibilities and must therefore be taken into account. To account for the aerodynamics the air drag characteristic coefficient in (4) can be modeled as

$$
\tilde{k}_{d}=k_{d}\left(1-\frac{\Phi(d)}{100}\right)
$$

where $\Phi(d)=-0.414 d+41.29$ and $0 \leq d \leq 99$ is the longitudinal relative distance between two vehicles. The linearized model for a HDV platoon is hence given by

$$
\dot{x}=A x+B u,
$$

where 


$$
A=\left[\begin{array}{ccccccccc}
\Theta_{1} & 0 & 0 & 0 & 0 & \cdots & 0 & 0 & 0 \\
1 & 0 & -1 & 0 & 0 & \cdots & 0 & 0 & 0 \\
0 & \delta_{2} & \Theta_{2} & 0 & 0 & \cdots & 0 & 0 & 0 \\
0 & 0 & 1 & 0 & -1 & \cdots & 0 & 0 & 0 \\
0 & 0 & 0 & \delta_{3} & \Theta_{3} & \cdots & 0 & 0 & 0 \\
\vdots & \vdots & \vdots & \vdots & \vdots & \ddots & \vdots & \vdots & \vdots \\
0 & 0 & 0 & 0 & 0 & \cdots & \Theta_{N-1} & 0 & 0 \\
0 & 0 & 0 & 0 & 0 & \cdots & 1 & 0 & -1 \\
0 & 0 & 0 & 0 & 0 & \cdots & 0 & \delta_{N} & \Theta_{N}
\end{array}\right],
$$$$
B=\left[\begin{array}{ccccc}
k_{e_{1}} & 0 & 0 & \cdots & 0 \\
0 & 0 & 0 & \cdots & 0 \\
0 & k_{e_{2}} & 0 & \cdots & 0 \\
0 & 0 & 0 & \cdots & 0 \\
0 & 0 & k_{e_{3}} & \cdots & 0 \\
\vdots & \vdots & \vdots & \ddots & \vdots \\
0 & 0 & 0 & \cdots & 0 \\
0 & 0 & 0 & \cdots & k_{e_{N}}
\end{array}\right], \quad x=\left[\begin{array}{c}
v_{1} \\
d_{12} \\
v_{2} \\
d_{23} \\
v_{3} \\
\vdots \\
v_{N-1} \\
d_{(N-1) N} \\
v_{N}
\end{array}\right]
$$

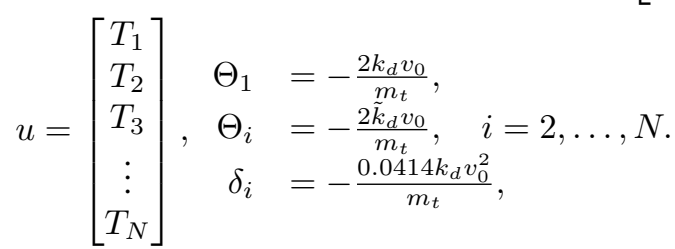

The system under consideration has a block tridiagonal structure on which the proposed controller design method can be implemented.

\section{Cost function}

In this section we propose a suitable set-up for the weight matrices $Q$ and $R$ based upon physical insight.

For general LQR-design the weighting factors need to be specified and adjusted based upon the results of the specified design goals. In the proposed decentralized control algorithm the weighting factors can be set separately for each subsystem. The lead vehicle's objective is to follow a given reference velocity and minimize the control input with respect to fuel optimality. However, the follower vehicles in the platoon have an additional objective of maintaining the set intermediate distance. The desired relative distance generally varies depending on the vehicle velocity. It is determined by setting a timegap $\tau \mathrm{s}$, which gives the desired headway as $d_{i j}=\tau v_{j}$. Thus, considering the platoon objectives, the cost function can be set up as

$$
\begin{array}{r}
J\left(T_{i}^{*}\right)=\min _{T_{i}} \int_{t_{0}}^{t_{f}} w_{i}^{\tau}\left(d_{(i-1) i}-\tau v_{i}\right)^{2}+w_{i}^{\Delta v}\left(v_{i-1}-v_{i}\right)^{2} \\
+w_{i}^{d} d_{(i-1) i}^{2}+w_{i}^{v} v_{i}^{2}+w_{i}^{T_{i}} T_{i}^{2} d t \\
=\min _{T_{i}} \int_{t_{0}}^{t_{f}}\left[\begin{array}{c}
v_{i-1} \\
d_{(i-1) i} \\
v_{i}
\end{array}\right]^{T} Q_{i}\left[\begin{array}{c}
v_{i-1} \\
d_{(i-1) i} \\
v_{i}
\end{array}\right]+R_{i} T_{i}^{2} d t
\end{array}
$$

where

$$
\begin{aligned}
Q_{i} & =\left[\begin{array}{ccc}
w_{i}^{\Delta v} & 0 & -w_{i}^{\Delta v} \\
0 & w_{i}^{d}+w_{i}^{\tau} & -\tau w_{i}^{\tau} \\
-w_{i}^{\Delta v} & -\tau w_{i}^{\tau} & \tau^{2} w_{i}^{\tau}+w_{i}^{\Delta v}+w_{i}^{v}
\end{array}\right], \\
R_{i} & =w_{i}^{T_{i}} .
\end{aligned}
$$

In accordance with the objective for a vehicle traveling in a platoon, $w_{i}^{\tau}$ in (6) determines the importance of not deviating from the desired time gap and $w_{i}^{\Delta v}$ creates a cost for deviating from the velocity of the preceding vehicle. The following terms, $w_{i}^{d}, w_{i}^{v}, w_{i}^{T_{i}}$, put a cost on the deviation from the linearized states and the control input. Since the main objective is to maintain a set intermediate distance, $w_{i}^{\tau}$ and $w_{i}^{\Delta v}$ must be set larger than the remaining weights.

\section{Robustness Evaluation}

In this section, we state a definition of string stability and give the performance by analyzing if the proposed controller produces a string stable system. An analytic expression is derived for the system under consideration and numerical results are given to show that the system is string stable.

Relative distance and velocity tracking are key factors in measuring the performance of the system. However, a concern regarding the robustness is frequently raised in vehicle platooning applications. In [19] a definition of string stability is presented. String stability can loosely be described as the ability to suppress a disturbance along the platoon. We will use a less rigorous approach similar to that presented in [20] and [21]. Assuming that the $i$ :th vehicle (Fig. 1) controls the headway distance by using only information from the immediate preceding vehicle, the transfer function from the lead vehicle's velocity $v_{1}$ to the tail-end vehicle's velocity $v_{n}$ can be expressed as

$$
V_{n}(s)=G_{1}^{v}(s) G_{2}^{v}(s) \cdots G_{n-1}^{v}(s) V_{1}(s)
$$

where

$$
V_{i}(s)=G_{i}^{v}(s) V_{i-1}(s), \quad i=2, \ldots, n .
$$

and $V(s):=\mathcal{L}(v(t))$ is the Laplace transform of the time domain velocity. We define that a string of $n$ vehicles in a platoon is string stable if for all $i=2, \ldots, n$

$$
\left\|G_{i}^{v}\right\|_{\infty} \leq 1
$$


where $\|\cdot\|_{\infty}$ indicates the maximum peak of the frequency response. The definition states that a deviation in the lead vehicles velocity from its steady-state value should not be amplified downstream.

The plant model (5) together with state-feedback

$$
u_{i}=-\left(L_{i}^{1} v_{i-1}+L_{i}^{2} d_{(i-1) i}+L_{i}^{3} v_{i}\right)
$$

gives the transfer function relation

$$
\begin{aligned}
V_{i}(s)= & \frac{\left(\delta_{i}-k_{e_{i}} L_{i}^{2}\right)}{s-\left(\Theta_{i}-k_{e_{i}} L_{i}^{3}\right)} D_{(i-1) i}(s) \\
& -\frac{k_{e_{i}} L_{i}^{1}}{s-\left(\Theta_{i}-k_{e_{i}} L_{i}^{3}\right)} V_{i-1}(s) .
\end{aligned}
$$

The transfer function for the relative distance is given by

$$
D_{(i-1) i}(s)=\left(V_{i-1}(s)-V_{i}(s)\right) / s .
$$

By combining (11) and (12) it is straight forward to derive the transfer functions

$$
V_{i}(s)=G_{i}^{v}(s) V_{i-1}(s)
$$

where

$$
G_{i}^{v}(s)=\frac{-k_{e_{i}} L_{i}^{1} s+\delta_{i}-k_{e_{i}} L_{i}^{2}}{s^{2}-\left(\Theta_{i}-k_{e_{i}} L_{i}^{3}\right) s+\delta_{i}-k_{e_{i}} L_{i}^{2}}
$$

As presented in Sec. V, we have considered a platoon consisting of $N=6$ identical vehicles and utilized equal LQR-weights for each follower vehicle. Thus, the transfer functions $G_{i}^{v}(s)$ are identical for each vehicle pair with $\Theta_{i}=-3.6 \times 10^{-3}, \delta_{i}=1.48 \times 10^{-5}, k_{e_{i}}=0.148 \times 10^{-3}$, $\forall i$. Hence, the maximum peak response for each transfer function can easily be calculated, (15), by inserting the subsystem LQR feedback gains $L_{1}=0.98 \times 10^{3}, L_{i}=$ $10^{3} \times[-6.69,-577.35,584.03]$, using Algorithm 1, into (14).

$$
\left\|G_{i}^{v}\right\|_{\infty}=1.00, i=2, \ldots, 6 .
$$

The results show that the robustness condition in (10) is satisfied. If additional HDVs are added to the platoon with identical weighting parameters $Q_{i}$ and $R_{i}$, the transfer function, (14), and inherently the maximum peak response, (15), will not change. Thus, the proposed decentralized controller design produces a string stable control regardless of how many vehicles of identical configuration that are added to the platoon.

\section{Simulations}

In this section, we evaluate the proposed controller algorithm by giving an example of how to derive the controllers for a system involving a HDV platoon consisting of six vehicles. The performance is evaluated through simulation results and we also investigate the feasibility and fuel efficiency of the derived controller.

When studying the behavior of vehicles within a finite platoon, the velocity does not deviate significantly from the lead vehicles velocity trajectory. The control strategy is simply to provide an input that maintains the platoon velocity at a set relative distance. However, concern arises when a disturbance is introduced to the system. The disturbance can be modeled as a deviation in the lead vehicles velocity.

The controller for each vehicle is designed with respect to the proposed Algorithm 1. The optimal feedback gain, $L_{11}$, for subsystem 1 is derived through (16) with $A_{11}=\Theta_{1}$, $B_{1}=k_{e_{1}}, Q_{1}=w_{1}^{v}$, and $R_{1}=w_{1}^{T_{1}}$.

$$
\begin{aligned}
& \min _{u_{1}} \int_{t_{0}}^{\infty} w_{1}^{v} v_{1}^{2}+R_{1} T_{1}^{2} d t \\
& \text { s. t. } \dot{v}_{1}=\Theta_{1} v_{1}+k_{e_{1}} T_{1},
\end{aligned}
$$

The the locally optimal feedback is given by $u_{1}^{*}=-L_{11} v_{1}+$ $l_{0} w$, where $l_{0}$ is the static feedback gain, and $w$ is the imposed disturbance. The controller for the rest of the subsystems in this case are derived iteratively in (17) with $A_{(i-1)(i-1)}=\left[\begin{array}{ll}\Theta_{i-1} & 0\end{array}\right], A_{i(i-1)}=\left[\begin{array}{ll}1 & 0 \\ 0 & \delta_{i}\end{array}\right], A_{i i}=\left[\begin{array}{c}-1 \\ \Theta_{i}\end{array}\right]$, $B_{i-1}=k_{e_{i-1}}, B_{i}=\left[\begin{array}{c}0 \\ k_{e_{i}}\end{array}\right], L_{i-1}=L_{i-1}^{3}$, and $Q_{i}, R_{i}$ given in (7).

$$
\begin{aligned}
& \min _{u_{i}} \int_{t_{0}}^{\infty}\left[\begin{array}{c}
v_{i-1} \\
d_{(i-1) i} \\
v_{i}
\end{array}\right]^{T} Q_{i}\left[\begin{array}{c}
v_{i-1} \\
d_{(i-1) i} \\
v_{i}
\end{array}\right]+R_{i} T_{i}^{2} d t \\
& \text { s.t. } \\
& {\left[\begin{array}{c}
\dot{v}_{i-1} \\
\dot{d}_{(i-1) i} \\
\dot{v}_{i}
\end{array}\right]=\left[\begin{array}{ccc}
\Theta_{i-1}-k_{e_{i-1}} L_{i-1}^{3} & 0 & 0 \\
1 & 0 & -1 \\
0 & \delta_{i} & \Theta_{i}
\end{array}\right]\left[\begin{array}{c}
v_{i-1} \\
d_{(i-1) i} \\
v_{i}
\end{array}\right]} \\
& +\left[\begin{array}{c}
0 \\
0 \\
k_{e_{i}}
\end{array}\right] T_{i}
\end{aligned}
$$

By utilizing $L_{i-1}^{3}$ in (17), which is the gain corresponding to the available state of the preceding vehicle's velocity, the controller becomes independent of all other indirectly preceding vehicles. The optimal feedback gain is obtained by solving the Riccati-equations for each subsystem as described in Algorithm 1. Hence the optimal control input is given as

$$
u_{i}^{*}=-\left[\begin{array}{lll}
L_{i}^{1} & L_{i}^{2} & L_{i}^{3}
\end{array}\right]\left[\begin{array}{c}
v_{i-1} \\
d_{(i-1) i} \\
v_{i}
\end{array}\right], i=2, \ldots, 6 .
$$

The modeled HDVs are described as traveling in a longitudinal direction on a flat road. The maximum engine and braking torque for a commercial HDV varies based upon vehicle configuration but can be approximated to be $3000 \mathrm{Nm}$ and $60000 \mathrm{Nm} /$ Axle respectively. The minimum time gap is $\tau=1 \mathrm{~s}$ and the mass of the vehicles are set to $m=40000 \mathrm{~kg}$, which is generally considered to be the standard weight of a long haulage heavy duty vehicle. 

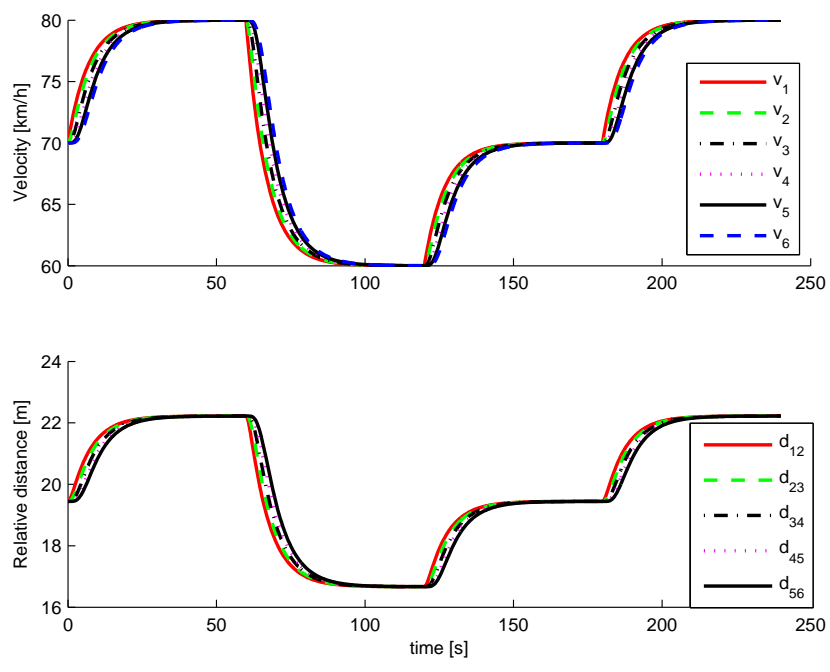

Fig. 2. The figure shows a platoon of $6 \mathrm{HDV}$, where a disturbance in velocity of the lead vehicle is imposed.

TABLE I

TABLE OF THE REQUIRED CONTROL INPUT (TORQUE) TO HANDLE THE DISTURBANCES IN FIG. 3.

\begin{tabular}{c|c|c|c|c|c|c}
$\mathrm{i}=$ & 1 & 2 & 3 & 4 & 5 & 6 \\
\hline$\left\|T_{i}\right\|_{2}[\mathrm{kNm}]$ & 45.5 & 42.1 & 40.8 & 39.8 & 39.0 & 38.4 \\
$T_{i}^{\text {max }}[\mathrm{kNm}]$ & 2.78 & 2.00 & 1.83 & 1.72 & 1.64 & 1.57 \\
$T_{i}^{\text {min }}[\mathrm{kNm}]$ & -5.49 & -3.95 & -3.61 & -3.39 & -3.23 & -3.10 \\
\hline
\end{tabular}

All the vehicles are assumed to be travelling in the steady state velocity $v_{0}=19.44 \mathrm{~m} / \mathrm{s}(70 \mathrm{~km} / \mathrm{h})$ and relative distance $d_{0}=\tau v_{0}$

Based upon these physical constraints, we investigate the controller performance when several disturbances are imposed on a $N=6$ vehicle platoon (Fig. 2). The disturbances can be explained by the following scenario. The lead vehicle is first forced to accelerate through a step input from $70 \mathrm{~km} / \mathrm{h}$ to $80 \mathrm{~km} / \mathrm{h}$ due to a new road speed point. When reaching $80 \mathrm{~km} / \mathrm{h}$ it suddenly has to decelerate to a lower speed of $60 \mathrm{~km} / \mathrm{h}$, because an obstruction in the form of a slower vehicle has entered the lane that has not yet reached the road speed. The obstructing vehicle increases its speed to $70 \mathrm{~km} / \mathrm{h}$ and then switches lanes, enabling the platoon to resume the road speed again.

The control design handles the disturbance well and demonstrates a good tracking performance. It can be seen (Fig. 2) that there is no overshoot in the velocity or relative distance tracking. The control input required (Fig. 3) to produce the tracking performance is also well within the boundaries of what is known to be physically obtainable. However, an engine cannot produce an instantaneous input torque. Therefore, a ramp input more suitable in these applications.

Table I shows the maximum, minimum, and accumulated torque energy that was required to account for the disturbances. The results show that the required input energy, which corresponds to the fuel consumption, decreases along

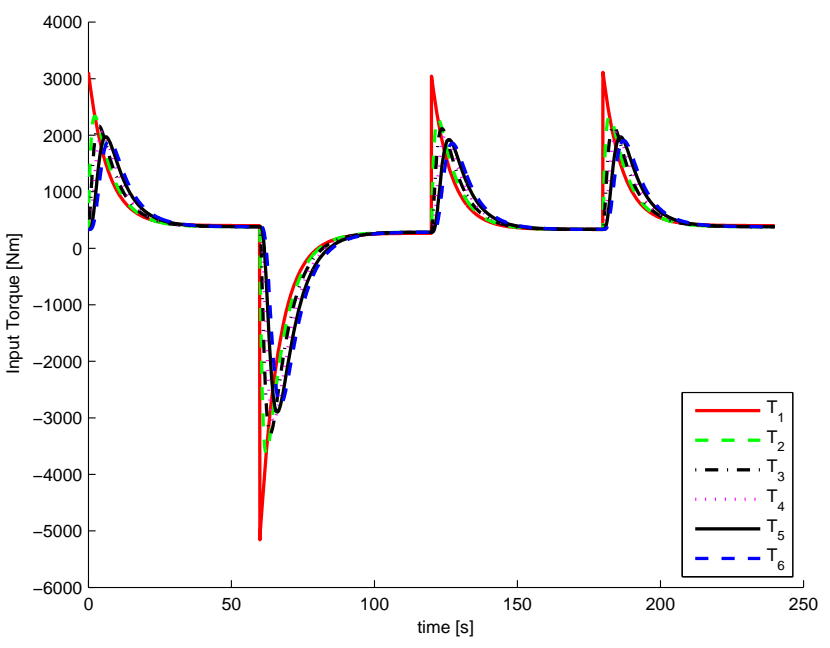

Fig. 3. The figure shows a the corresponding input torque for the platoon of 6 HDVs, where a disturbance in velocity of the lead vehicle is imposed.

the chain of vehicles. Hence, the designed controller is fuel efficient. In comparison with a centralized controller for an identical scenario, assuming that all states are available at all time instances, the control effort energy is up to $29 \%$ higher for the decentralized controller. However, the decentralized control system have a $41 \%$ lower rise time because the follower vehicle dynamics are not taken into consideration. Hence, there is a trade-off in system performance. In freight transportation, the delivery time is equally important as fuel consumption.

\section{SUMMARY AND CONCLUSIONS}

The proposed decentralized controller in this paper addresses the basic communication topology and handles dynamically interconnected systems. It can easily be extended to more advanced communication topologies such as receiving state information from additional preceding or follower vehicles. However, extending the communication topology will increase the controller and computational complexity, which is restricted in many real life applications. The proposed methodology produces a simple and energy efficient suboptimal decentralized controller with good tracking performance, stability, and robustness properties. It is simple in its nature, since the optimal control input, is calculated sequentially for each vehicle and is only based on information from the preceding vehicle. Thus, it is also scalable, since adding a vehicle to the end of the chain will not mandate a change in decentralized controllers within the platoon. Yet it maintains the overall system performance. A centralized control strategy might produce a lower LQR-cost, however it is not realistic to assume that an agent in the platoon would know the state of all the other agents in the formation at any given time and be able to use it to calculate the control input due to physical constraints in the information flow. Hence, the control design methodology can most likely be implemented in real life applications. 
On the other hand, as the control law is based on a linearized model, it is interesting to evaluate it on the actual HDV dynamics. In real life applications many parameter uncertainties and nonlinearities exist. For example, the braking power becomes nonlinear due to a temperature variation in the braking hardware and the produced engine torque transferred to the wheels is a nonlinear function of the current gear. If nonlinearities are taken into account, a more fuel efficient control strategy could most likely be produced. Also, delays or losses within the communication is a common occurrence in real applications. Robustness, in the sense of string stability, is only guaranteed for identical vehicles and LQR weighting parameters. It is interesting to determine necessary and sufficient conditions for heterogeneous platoons such that robustness in a finite vehicle HDV platoon is always guaranteed. Hence, these issues follows as a natural extension and future work within this subject.

\section{ACKNOWLEDGMENTS}

The authors would like to thank Gustav Hammar and Vadim Ovtchinnikov for their contribution to part of the examples presented in this work.

\section{REFERENCES}

[1] H. Wolf-Heinrich and S. R. Ahmed, Aerodynamics of Road Vehicles. Warrendale: Society of Automotive Engineers, Inc, 1998.

[2] A. A. Alam, A. Gattami, and K. H. Johansson, "An experimental study on the fuel reduction potential of heavy duty vehicle platooning," 13th International IEEE Conference on Intelligent Transportation Systems, September 2010.

[3] J. Marshak, "Elements for a theory of teams," Management Sci., pp. 1:127-137, 1955.

[4] D. Q. Mayne, "Sequential design of linear multivariable systems," in Proc. IEE D, Control Theory \& Appl., vol. 126, no. 6, 1979, pp. 559563.

[5] B. Bamieh, F. Paganini, and M. A. Dahleh, "Distributed control of spatially invariant systems," IEEE Transactions on Automatic Control, vol. 47, no. 7, July 2002.

[6] R. D'Andrea, "A linear matrix inequality approach to decentralized control of distributed parameter systems," in American Control Conference, 1998. Proceedings of the 1998, vol. 3, June 1998, pp. 1350 $-1354$.
[7] O. Khorsand and A. Gattami, "A decentralized stabilization scheme for a class of large-scale interconnected systems," in 18th IFAC World Congress, Milano, Italy, August 2011.

[8] B. Bamieh, M. R. Jovanović, P. Mitra, and S. Patterson, "Effect of topological dimension on rigidity of vehicle formations: Fundamental limitations of local feedback," IEEE Conference on Decision and Control, December 2008.

[9] P. Barooah and J. ao P. Hespanha, "Error amplification and disturbance propagation in vehicle strings with decentralized linear control," in 44th IEEE Conference on Decision and Control, and the European Control Conference, December 2005, pp. 1350 - 1354.

[10] J. Rogge and D. Aeyels, "Decentralized control of vehicle platoons with interconnection possessing ring topology," 44th IEEE Conference on Decision and Control, and the European Control Conference, pp. 1491-1496, 2005.

[11] B. Bamieh and M. R. Jovanović, "On the ill-posedness of certain vehicular platoon control problem," IEEE Transactions on Automatic Control, vol. 50, no. 9, September 2005.

[12] S. Sudin and P. A. Cook, "Two-vehicle look-ahead convoy control systems," 59th IEEE Vehicular Technology Conference, VTC, vol. 5, pp. $2935-2939,2004$.

[13] D. Swaroop and J. Hedrick, "String stability of interconnected systems," IEEE Transactions on Automatic Control, 1996.

[14] P. Varaiya, "Smart cars on smart roads: Problem of control," IEEE Transactions on Automatic Control, vol. 38, NO. 2, February 1993.

[15] W. Levine and M. Athans, "On the optimal error regulation of a string of moving vehicles," IEEE Transactions on Autmatic Control, vol. AC11, no.3, pp. 355-361, 1966.

[16] S. S. Stanković, M. J. Stanojević, and D. D. Šiljak, "Decentralized overlapping control of a platoon of vehicles," IEEE Transactions on Control Systems Technology In Control Systems Technology, vol. 8, No.5, pp. $816-832,2000$.

[17] W. B. Dunbar and R. M. Murray, "Receding horizon control of multivehicle formations: a distributed implementation," Automatica, vol. 42, pp. $549-558,2006$.

[18] P. Sahlholm and K. H. Johansson, "Road grade estimation for lookahead vehicle control using multiple measurement runs," Control Engineering Practice, vol. 18, no. 11, pp. 1328 - 1341, 2010.

[19] D. Swaroop and J. K. Hedrick, "String stability of interconnected systems," IEEE Transactions on Automatic Control, vol. 41, no. 3, March 1996.

[20] S. Sheikholeslam and C. A. Desoer, "Longitudinal control of a platoon of vehicles with no communication of lead vehicle information: A system level study," IEEE Transactions on vehicular technology, vol. 42, no. 4, November 1993.

[21] Y. Yamamura and Y. Seto, "A study of string-stable acc using vehicleto-vehicle communication," SAE World Congress, 2006, 2006-010348 . 